Results 12 patients satisfied inclusion criteria in cycle one and 6 in cycle two. Data demonstrated improvements in a number of areas. These included involvement of the palliative care team increasing from $50 \%$ to $83 \%$, documentation of plans for hydration from $42 \%$ to $67 \%$ and nutrition from $50 \%$ to $67 \%$, rationalisation of medication from $42 \%$ to $83 \%$ and of interventions from $33 \%$ to $83 \%$. Improvement was seen in documentation of communication with patients and their family such as those relating to prognosis from $75 \%$ to $100 \%$, CPR decisions from $50 \%$ to $83 \%$, preferred place of death from $25 \%$ to $83 \%$, assessment of Psychosocial or spiritual needs of the patient from $50 \%$ to $83 \%$ and for their families from $33 \%$ to $83 \%$. However, the percentage of patients with CPR decisions remained $83 \%$ in both cycles.

Conclusions Use of simple educational interventions and prompts have demonstrated improved documentation of individualised end of life care plans in ASU/Trauma. Ongoing audit and continued education may lead to sustainable improvement in the quality of EOLC delivered by these teams.

\section{ASSESSING THE END OF LIFE CARE NEEDS OF PATIENTS IN ACUTE HOSPITAL SETTING? FINDINGS FROM A SCOPING REVIEW}

Mary Pocock, Cara Bailey, Alistair Hewison. University of Birmingham

\subsection{6/spcare-2020-PCC.98}

Background High quality end-of-life care is required to ensure the care patients receive in acute settings meets their needs, but end-of-life care needs are not always adequately assessed on admission. The use of appropriate assessment tools that guide clinical decision-making and care planning could improve care and enable rapid transfer to the preferred place of care. This paper reports the findings of a literature review which investigates the use of end-of-life care assessment tools in acute hospitals.

Methods This scoping review adopted a three-step search strategy using five databases. 760 citations were generated and following the PRISMA process, 55 full texts were assessed for eligibility and sixteen of these were identified as suitable for data synthesis. Data were retrieved from each paper and analysed thematically using Braun and Clark (2006). The assessment tools were critiqued to assess validity and reliability to determine which were the most feasible for use in the acute hospital setting. A further search was carried out to identify any reports of the use of the tools in clinical practice.

Results Sixteen papers published since 2000, reported the development and application of fifteen different assessment tools in acute hospitals. Of these, only five reported the use of assessment tools in acute clinical practice. Analysis identified four key issues - potential improvement to patient wellbeing, training on the usability of assessment tools, burden to patient and staff, and validity and reliability of the assessment tools.

Conclusions Only a limited number of end of life assessment tools have been developed and fewer have been tested in routine practice. Some of the assessment tools reported for use in the acute hospitals made a positive impact on patients' end-oflife care. Further research is necessary to understand how assessment tools can help assess end-of-life care needs and ultimately improve supportive care in the acute setting.

\section{9 \\ IMPROVING DNAR DISCUSSIONS: A QUALITATIVE QUALITY IMPROVEMENT PROJECT}

Ruth C Porther, Charles A Haywood Smith. Aneurin Bevan Health Board

\subsection{6/spcare-2020-PCC.99}

Background Patients often do not recall all the key points in discussion with healthcare professionals, perhaps even more so when discussing topics like a Do Not Resuscitate (DNAR) decision. If communication in these potentially emotional situations can be enhanced using patient leaflets compared to discussion alone, perhaps it would be worth considering using them more often in future. The aim of this project was to ascertain whether at least $50 \%$ of participants felt that using the All Wales patient leaflets improved their understanding of CPR and DNAR decisions.

Method 10 patients and loved ones who were present for the initial DNAR discussion were surveyed. A questionnaire elicited whether they felt their understanding of CPR and DNAR decisions was good before reading the leaflet and if it improved after. This involved 8 participants on the Medical Assessment Unit at the Royal Gwent Hospital and 2 on a Care of the Elderly ward at Nevill Hall Hospital who had documented DNAR discussions.

Results 50\% felt the leaflet improved their understanding and that it would have benefited them at the time of discussion. $70 \%$ felt it was a useful reference and $40 \%$ asked to keep the leaflet after the encounter. Anecdotally, cases where participants particularly found it useful were those where death was not imminent, large numbers of family members were involved and had different views and in complex advance care planning

Conclusion The data gathered from this small, qualitative project suggest there may be a role for patient information leaflets on DNAR decisions when used in the right context.

\section{CARE DOESN'T STOP WHEN LIFE DOES: IMPROVING DOCUMENTATION OF CARE AFTER DEATH IN A HOSPITAL SETTING}

Samuel Raveney, Rina Patel, Jackie Bebb. Epsom and St Helier University Hospitals NHS Trust

\subsection{6/spcare-2020-PCC.100}

Background Staff responsible for care after death should 'Record all aspects of care after death in locally relevant documentation and identify the professionals involved.' ${ }^{\text {A }}$ Trust-wide audit in 2017 showed that documentation of care after death at the trust was poor and only $30 \%$ of patients had any documentation of care after death recorded. The care after death checklist was developed and launched as a prompt for staff providing care after death to complete.

Aims To measure and evaluate the correct use of the care after death checklist and to ascertain whether the checklist has effected the documentation of the care provided after death.

Methods A retrospective audit involving review of the clinical notes and the care after death Checklist when completed was performed. Thirty sets of notes, across two hospitals within the trust were reviewed. Patients who died in the first two 
weeks of April and May 2019 and were over 18 years old were included.

Results The care after death checklist was used in 27 out of the 30 patient notes audited. There was documentation of care after death in $100 \%$ of patients using the checklist compared to $67 \%$ in the cases without using the checklist. There was superior quality of information documented when the checklist was used compared to when it was not used across multiple domains. For example, in 100\% of cases where the checklist was used there was documentation of explanation of the procedure for collecting the death certificate to relatives, compared to $0 \%$ when the checklist was not used.

Conclusion The use of a standardised checklist improves the quality and breadth of documentation of care after death provided in a hospital setting.

\section{REFERENCES}

1. The Care After Death: Guidance for staff responsible for care after death, 2nd Edition. Published 2015. https://www.hospiceuk.org/what-we-offer/clinical-andcare-support/clinical-resources

\section{USE OF STEROIDS IN PALLIATIVE CARE}

Amanda Richardson, Emma McDougall, Katherine E Frew. Northumbria Healthcare Foundation Trust

\subsection{6/spcare-2020-PCC.101}

Background Steroids are frequently prescribed in palliative care for several indications, often concurrently. Identifying the original indication and thus a safe withdrawal regimen is, however, not straightforward as patients transition across different clinical settings. We conducted this Quality Improvement Project to assess current practice of steroid prescribing and titration in dying patients on an NHS Palliative Care Unit in Northumbria Healthcare NHS FT.

Methods All patients who were prescribed steroids between January and April 2019 were included in the study, and followed through until discharge or death. Details of the indication, dose, and the documented plan for dose reduction and cessation were collected.

Results 43 patients were prescribed steroids: all of these were given dexamethasone. 17 patients died in the period under study; the remainder were discharged. While $48 \%$ had a plan for reduction of steroids documented, this was inconsistent and highly variable.

The mean number of days patients remained on dexamethasone was 19.2 (range 3-54 days). The most common reason for stopping steroids was patient deterioration or death (40\%). $88 \%$ of patients who died, were given dexamethasone subcutaneously in the last days of life.

Discussion Steroid use can lead to significant morbidity, with adverse effects reported in up to $63 \%$ of prescriptions. Adrenal suppression can occur when steroids are used for over 3 weeks. It is critical to be able to establish the indication for and the duration of steroid use, in order to prevent unnecessary harm by the continuation of a subcutaneous injection even into the last days of life. This is the start of a plan, do, study, act cycle. The next iteration of this introduces mandatory documentation of the indication for use within electronic prescribing modules.

\section{ANTICIPATORY PRESCRIBING IN COMMUNITY END OF LIFE CARE IN THE UK: A MIXED-METHODS STUDY OF HEALTHCARE PROFESSIONALS' VIEWS CONCERNING BEST PRACTICE AND AREAS IN NEED OF IMPROVEMENT}

Richella Ryan, Anna Spathis, Ben Bowers, Mila Petrova, Sarah Hopkins, Tessa Morgan, Markus Schichtel, Brooke Swash, Louisa Polak, Arjun Kingdon, Stephen Barclay. University of Cambridge

\subsection{6/spcare-2020-PCC.102}

Introduction Anticipatory prescribing (AP), the supply of injectable medication to a patient in advance of need, is wellestablished practice in the UK although there is currently limited evidence and guidance to support best practice. With a view to developing guidance, we explored the views of UK healthcare professionals (HCPs) about best practice and areas in need of improvement in AP.

Methods Two day workshops were held in London and Cambridge, attended by 89 delegates. Participants completed an initial survey and then participated in two focus groups at the end of which they wrote down 3 'top-tips' for:

1. achieving best practice and

2. areas in need of improvement concerning AP. Analysis involved descriptive statistics of survey responses and thematic analysis of free-text 'top-tips'.

Results $71 / 89(80 \%)$ of participants completed the survey: 25 (35\%) palliative care nurses, 24 (33\%) palliative medicine consultants, 22 (30\%) 'other' (GPs, community nurses, pharmacists). $76 \%$ had $>10$ years of experience and $75 \%$ were involved in AP a few times each week. On a rating scale of 1-5 (1=low, 5=high), 38\% were confident (rating 4) that AP is done well [median: 3(IQR: 3-4)] and 20\% were concerned (rating 4) about unsafe practice in AP [median=3 (IQR: 2-3)]. $89 \%$ agreed (score 4 ) that more guidance is needed to support AP [median=4 (IQR: 4-5)]. Top-tips for improving AP were: 1) improving communication with patients and families and between HCPs, 2) increasing out-of-hours access to medications, 3) supporting staff with training, education and guidance, and 4) reducing cross-system complexity by unifying documents and IT systems.

Conclusions There is a high demand amongst HCPs for unified guidance and documentation to support AP. A national guideline development group is being formed in response to this and at the request of NHS England. In-depth analysis of focus group transcripts is underway.

\section{IT'S HIGH TIME FOR STRAIGHT ANSWERS ABOUT CANNABIS: RESULTS FROM A SURVEY OF HEALTHCARE PROFESSIONALS WORKING IN ONCOLOGY AND HAEMATOLOGY IN A UNIVERSITY HOSPITAL IN ENGLAND}

Rachelle Schofield, Alice Tew, Jon Tomas. University of Birmingham, Queen Elizabeth Hospital Birmingham

\subsection{6/spcare-2020-PCC.103}

Introduction Since 2018 the prescription of unlicensed cannabis-based products (CBPs) has been legal in the UK in certain indications by certain medical professionals. Despite NHS England guidance it is not generally known how often Healthcare 